\title{
Avaliação de modelos de estimativa do saldo de radiação e do método de Priestley-Taylor para a região de Dourados, MS
}

\author{
Carlos R. Fietz $^{1} \&$ Gilberto F. Fisch $^{2}$
}

\begin{abstract}
RESUMO
Este trabalho foi realizado com o objetivo de avaliar modelos de estimativa da radiação líquida e o método de Priestley-Taylor para a região de Dourados. 0 ajuste dos parâmetros dos modelos foi realizado com base em uma série de 1.421 dados diários de radiação líquida, radiação solar global, radiação extraterrestre, temperaturas máxima e mínima. U ma segunda série de dados com 360 observações diárias foi utilizada para validar as equações geradas. A evapotranspiração de referência $\left(\mathrm{ET}_{0}\right)$ foi estimada pela equação de Priestley-Taylor, como função da radiação solar global. Os valores de $E_{0}$ foram comparados com 218 medidas lisimétricas. As estimativas de radiação líquida geradas com base apenas nas temperaturas máximas e mínimas não foram satisfatórias, mas o modelo que utilizou radiação extraterrestre, além dessas duas variáveis, apresentou performance mediana. Os modelos que utilizaram a radiação solar gl obal como variável independente tiveram desempenho classificados como ótimos. 0 método de Prietley-Taylor apresentou desempenho muito bom, possibilitando estimar a evapotranspiração de referência diária da região de Dourados com base na radiação solar global e na temperatura média do ar.
\end{abstract}

Palavras-chave: radiação líquida, radiação solar global, evapotranspiração de referência

\section{Evaluation of models to estimate net radiation and the Priestley-Taylor method in the region of Dourados, MS, Brazil}

\begin{abstract}
The objective of this work was to evaluate models to estimate net radiation and the Priestley-Taylor method in Dourados, in the State of M ato Grosso do Sul, Brazil. For the purpose, 1,421 daily observations of net radiation, global radiation, extraterrestrial radiation, and maximum and minimum air temperatures were used. Another database containing 360 of these same daily variables was used to independently test the models The reference evapotranspiration $\left(\mathrm{ET}_{0}\right)$ was estimated by the Priestley-Taylor method as a function of global radiation. The estimated values of ETo were compared with 218 lysimeter data. The equation based only on the maximum and minimum air temperatures showed unsatisfactory performance. A mean performance was obtained with the model based on the extraterrestrial radiation and the maximum and minimum air temperatures. The models which included global radiation as independent variable had a more satisfactory performance. The Prietley-Taylor method showed very good performance and can be used to estimate the daily reference evapotranspiration in the region of Dourados from the solar radiation and the mean air temperature.
\end{abstract}

Key words: net radiation, solar radiation, reference evapotranspiration

${ }^{1}$ Embrapa Agropecuária 0 este, CP 661, CEP 79804-970, Dourados, MS. Fone: (67) 3425-5122. E-mail: fietz@cpao.embrapa.br

${ }^{2}$ Centro Técnico Aeroespacial, Praça Marechal Eduardo Gomes, 50, CEP 12228-904, São José dos Campos, SP. E-mail: gfisch@iae.cta.br 


\section{INTRODUÇÃO}

O saldo de radiação ou de energia representa a quantidade de energia que está disponível para os processos de evapotranspiração, fotossíntese e de aquecimento do ar e do solo. Esta componente do balanço de energia é constituída predominantemente por radiação de ondas curtas e longas. O saldo de radiação depende fortemente do fluxo de radiação solar e em geral é positivo durante o dia (representando uma entrada maior do que a saída de energia, com o aquecimento do sistema solo-planta-atmosfera) e negativo à noite. Considerando a integração diária, usualmente o saldo de radiação é positivo (Sentelhas \& Nascimento, 2003).

A maior parte das estações meteorológicas do Brasil não dispõe de equipamentos para medida do saldo de radiação, pelo fato dos saldo-radiômetros apresentarem custo elevado e dificuldade de manutenção. Em virtude disto, o saldo de radiação geralmente é estimado. Em um estudo realizado em área de pastagem na Amazônia, Galvão \& Fisch (2000) avaliaram algumas fórmulas empíricas para estimar o saldo da radiação de ondas longas provenientes da atmosfera, um dos componentes do saldo de radiação de difícil medição. Recentemente, von Randow \& Alvalá (2006) aplicaram a mesma metodologia para a região do Pantanal.

A metodologia padrão para estimar o saldo de radiação é a recomendada pela FAO (Allen et al., 1998); no entanto, este método apresenta algumas dificuldades, principalmente na estimativa do saldo de radiação de ondas longas.

O saldo de radiação é o elemento meteorológico que exerce a maior influência na evapotranspiração. De maneira geral, não havendo restrição hídrica, quanto maior a disponibilidade de energia solar e conseqüentemente do saldo de radiação, maior também será a evapotranspiração. Considerando esse princípio, Priestley \& Taylor (1972) desenvolveram um método de estimava da evapotranspiração de referência que pode ser interpretado não só como uma versão do balanço de energia, mas também uma simplificação do método de Penman (Pereira et al., 1997), em que permanece apenas o termo radiativo corrigido por um coeficiente empírico de ajuste $(\alpha)$. O coeficiente a incorpora a energia adicional ao processo de evapotranspiração e varia com o tipo de superfície e condições de umidade (Sentelhas et al., 2000). O modelo de Priestley-Taylor foi desenvolvido com base em dados coletados em superfícies de água livre e em solos vegetados e desnudos. Nessa situação, os valores $\alpha$ variaram de 1,08 a 1,34, com valor médio de 1,26, considerado como a melhor estimativa. Sentelhas et al. (2000) verificaram que o valor mais apropriado de a para Piracicaba (SP) em períodos chuvosos foi significativamente inferior ao proposto por Priestley \& Taylor (1972), enquanto em períodos secos o valor foi bem próximo.

Considerando a importância do saldo de radiação e da evapotranspiração no sistema solo-planta-atmosfera, realizouse este trabalho, cujo objetivo foi avaliar modelos de estimativa do saldo de radiação e o método de Priestley-Taylor para a região de Dourados.

\section{MATERIAL E MÉTODOS}

Os dados meteorológicos foram coletados em uma estação meteorológica automática, instalada na Embrapa Agropecuária Oeste em Dourados, MS, cujas coordenadas geográfi-

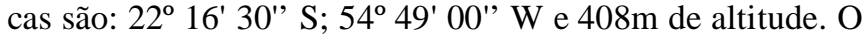
clima da região da Dourados é do tipo Cwa (clima mesotérmico úmido, verões quentes e invernos secos), com a temperatura do mês mais frio inferior a $18^{\circ} \mathrm{C}$ e a do mês mais quente superior a $22^{\circ} \mathrm{C}$ (Fietz \& Fisch, 2008).

A estação meteorológica possuía um sistema de aquisição de dados (Campbell Scientificic. CR10X, Logan, Utah, EUA) e os seguintes sensores: saldo de radiação (Q-7 net radiometer, Campbell Scientificic. Inc, Logan, Utah, EUA), radiação global (CM3 pyranometer, Kipp \& Zonen B.V., Delft, The Netherlands) e temperatura do ar (HMP45C temperature and relative humidity probe, Vaisala, Vanta, Finland).

Quatro tipos de equações para estimativa do saldo de radiação foram avaliados:

$$
\begin{gathered}
\mathrm{Rn}=\alpha \mathrm{TM}-\beta \mathrm{Tm} \\
\mathrm{Rn}=\alpha(\mathrm{TM}-\mathrm{Tm})^{\beta} \mathrm{Ra} \\
\mathrm{Rn}=\alpha \mathrm{Rs} \\
\mathrm{Rn}=\alpha \mathrm{Rs}+\beta \mathrm{Ra}
\end{gathered}
$$

em que Rn é o saldo de radiação $\left(\mathrm{MJ} \mathrm{m}^{-2} \mathrm{dia}^{-1}\right)$; TM é a temperatura máxima $\left({ }^{\circ} \mathrm{C}\right)$; Tm é a temperatura mínima $\left({ }^{\circ} \mathrm{C}\right)$; Ra é a radiação extraterrestre $\left(\mathrm{MJ} \mathrm{m}^{-2} \mathrm{dia}^{-1}\right)$, Rs é a radiação solar global ( $\mathrm{MJ} \mathrm{m}^{-2} \mathrm{dia}^{-1}$ ) e $\alpha$ e $\beta$ são os parâmetros das equações.

Realizou-se o ajuste dos parâmetros dos modelos com base em uma série de 1.421 dados diários de 01/01/2001 a $31 / 12 / 2005$. A radiação extraterrestre desse mesmo período foi calculada com base na latitude e na época do ano, através das expressões citadas por Allen et al. (1998). Uma segunda série de dados, coletada durante o ano de 2006, com 360 observações diárias dos mesmos elementos climáticos, foi utilizada para validar as equações geradas. Os parâmetros dos modelos foram estimados utilizando-se a técnica de ajuste não linear, segundo o critério dos mínimos quadrados (Daley, 1991).

Os valores estimados de saldo de radiação foram comparados com os medidos. Avaliou-se o desempenho dos modelos de estimativa pelo índice de confiança "c" proposto por Camargo \& Sentelhas (1997), obtido pelo produto do coeficiente de correlação (r) e o índice de concordância de Willmott (d) (Willmott, 1981):

$$
c=r . d
$$

A evapotranspiração de referência $\left(\mathrm{ET}_{0}, \mathrm{em} \mathrm{mm} \mathrm{dia}^{-1}\right)$ foi calculada pelo método Priestley \& Taylor (1972):

$$
\lambda \mathrm{ET}_{0}=\alpha \mathrm{W}(\mathrm{Rn}-\mathrm{G})
$$

em que $\alpha$ é o parâmetro Priestley-Taylor; Rn a radiação líquida $\left(\mathrm{MJ} \mathrm{m}^{-2} \mathrm{dia}^{-1}\right)$; G é o fluxo de calor do solo $\left(\mathrm{MJ} \mathrm{m}^{-2} \mathrm{dia}^{-1}\right)$; 
$\lambda$ é o calor latente de evaporação $\left(2,45 \mathrm{MJ} \mathrm{kg}^{-1}\right) \mathrm{e} \mathrm{W}$ é o fator de ponderação dependente da temperatura do ar ( $\mathrm{T}$, em $\left.{ }^{\circ} \mathrm{C}\right)$ e da constante psicrométrica, citado por Pereira et al. (2002):

$$
\begin{gathered}
\mathrm{W}=0,407+0,0145 \mathrm{~T} \quad\left(0^{\circ} \mathrm{C}<\mathrm{T} \leq 16^{\circ} \mathrm{C}\right) \\
\mathrm{W}=0,483+0,01 \mathrm{~T} \quad\left(16^{\circ} \mathrm{C}<\mathrm{T}<32^{\circ} \mathrm{C}\right)
\end{gathered}
$$

No entanto, um modelo de estimativa do saldo de radiação foi selecionado visando apresentar a Eq. 6 como função da radiação solar global (Rs). Nesse procedimento, utilizouse $\alpha=1,26$, valor médio para diversas coberturas, e não foi considerado o fluxo de calor do solo (G).

Os valores estimados de evapotranspiração de referência $\left(\mathrm{ET}_{0}\right)$ foram comparados com 218 medidas lisimétricas obtidas por Fietz et al. (2005), sendo o desempenho das estimativas de $\mathrm{ET}_{0}$ também avaliado pelo índice de confiança "c".

\section{RESULTADOS E DISCUSSÃO}

O modelo desenvolvido com base na temperatura máxima e temperatura mínima (Eq. 1) teve um desempenho classificado como péssimo (Tabela 1), apresentando um índice "c" de apenas 0,39 . Observou-se também que o coeficiente de variação dos dados gerados por esse modelo (16\%) foi bem menor que o dos valores medidos de saldo de radiação (38\%), comportamento que também pode ser visualizado na Figura 1A, pela maior dispersão dos pontos ao longo da reta 1:1.

Tabela 1. Desempenho dos métodos de estimativa do saldo de radiação $(R n)$ com base no coeficiente de determinação $\left(R^{2}\right)$, índice de concordância de Willmott (d) e índice "c"

\begin{tabular}{lcccccc}
\hline \multicolumn{1}{c}{ Determinação* } & Equação & $\begin{array}{c}\text { Rn média } \\
\left(\mathbf{M J} \mathbf{~ m}^{-2}\right. \\
\text { dia }^{-1} \text { ) }\end{array}$ & $\mathbf{C V}(\%)$ & $\mathbf{R}^{2}$ & $\mathbf{d}$ & $\mathbf{C}$ \\
Saldo-radiômetro & & 10,9 & 38 & & & \\
$\mathrm{Rn}=0,466 \mathrm{TM}-0,164 \mathrm{TM}$ & $(1)$ & 11,1 & 16 & 0,38 & 0,63 & 0,39 \\
$\mathrm{Rn}=0,063(\mathrm{TM}-\mathrm{Tm}) 0,661 \mathrm{Ra}$ & $(2)$ & 11.0 & 25 & 0,58 & 0,83 & 0,63 \\
$\mathrm{Rn}=0,435 \mathrm{Rs}$ & $(3)$ & 10,9 & 37 & 0,93 & 0,98 & 0,94 \\
$\mathrm{Rn}=0,401 \mathrm{Rs}+0,027 \mathrm{Ra}$ & $(4)$ & 11,0 & 35 & 0,94 & 0,98 & 0,95 \\
\hline
\end{tabular}

${ }^{*} \mathrm{TM}$ - temperatura máxima $\left({ }^{\circ} \mathrm{C}\right), \mathrm{Tm}$ - temperatura mínima $\left({ }^{\circ} \mathrm{C}\right), \mathrm{Ra}-$ radiação extraterrestre $\left(\mathrm{MJ} \mathrm{m}^{-2} \mathrm{dia}^{-1}\right)$ e Rs - radiação solar global $\left(\mathrm{MJ} \mathrm{m}^{-}\right.$ $\left.{ }^{2} \mathrm{dia}^{-1}\right)$

As estimativas de Rn em função da temperatura máxima (TM), temperatura mínima (Tm) e radiação extraterrestre (Ra) obtidas pela Eq.2, apresentaram desempenho mediano, com um índice "c" de 0,63 (Tabela 1). A melhor performance deste modelo, em relação à Eq.1, pode ser atribuída à inserção da variável Ra que, de certa maneira, modula a quantidade de energia que ficará disponível para os processos de evapotranspiração. Zanetti et al. (2004, 2006) avaliando Rn em função de TM, Tm e Ra, obtiveram um ajuste melhor para as condições de Campos de Goytacazes, RJ; no entanto, neste trabalho a Rn não foi medida mas estimada pela metodologia apresenta-
A.

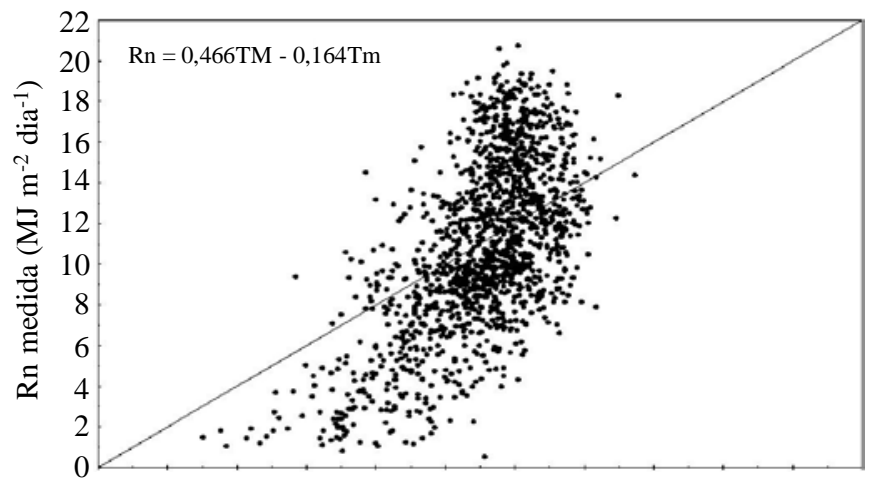

B.

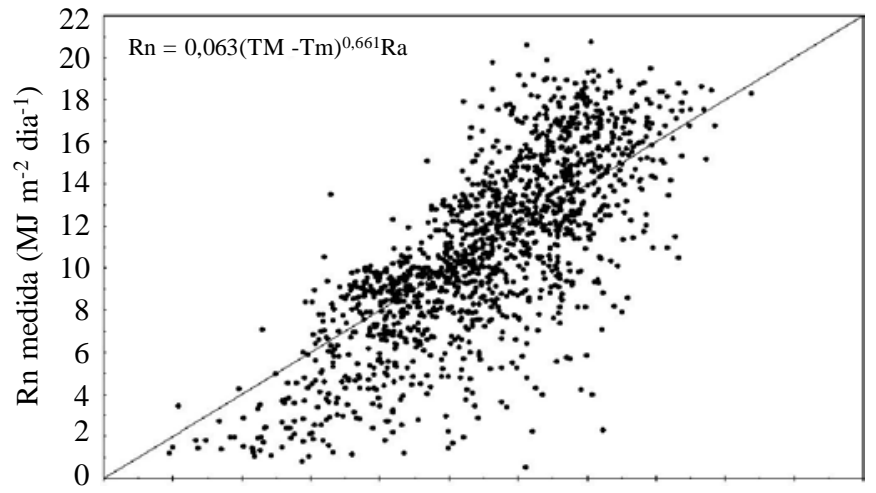

C.
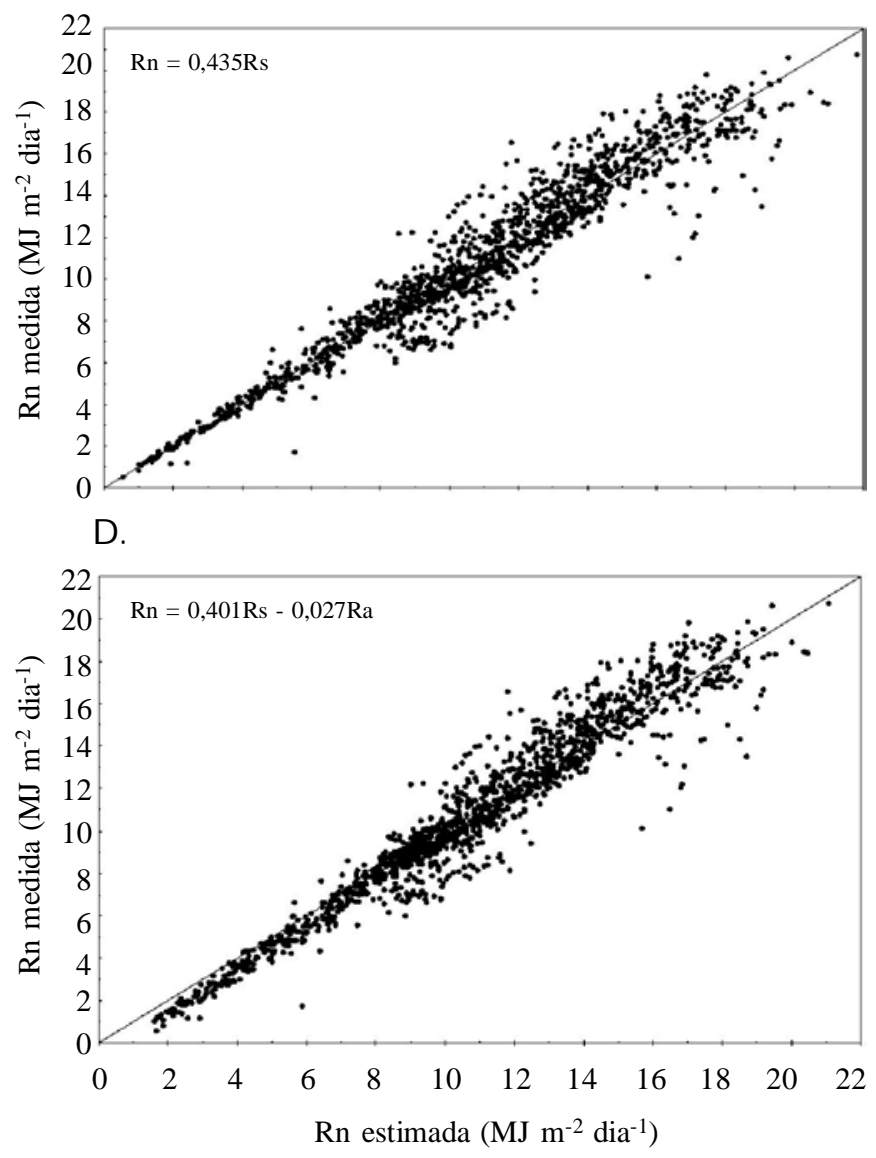

Figura 1. Relação entre saldo de radiação $(\mathrm{Rn})$ medida e estimada com base na temperatura máxima (TM), temperatura mínima (Tm), radiação solar global (Rs) e extraterrestre $(\mathrm{Ra})$ 
da no Boletim 56 da FAO (Allen et al., 1998). Além disso, em Campos dos Goytacazes a influência é mais marítimica, enquanto que em Dourados é mais continental.

O modelo que utilizou unicamente dados de radiação solar global como variável dependente (Eq. 3) mostrou desempenho ótimo, comportamento expresso pelo valor do índice "c" de 0,94 (Tabela 1) e pela pequena dispersão dos pontos ao longo da reta 1:1 (Figura 1C). O coeficiente de variação dos dados gerados pela Eq. 3 foi praticamente igual ao dos valores medidos de radiação líquida (Tabela 1), o que pode ser visualizado pela pequena dispersão dos pontos ao longo de toda a reta 1:1 (Figura 1C). Para as condições de Piracicaba, SP, Pereira et al. (1998) também obtiveram excelente ajuste com esse tipo de equação, concluindo que para aquele local o saldo de radiação pode ser representado por $57 \%$ da radiação solar global. No caso de Dourados, esta razão foi de $44 \%$.

A Eq.4, que estima o saldo de radiação com a introdução da variável radiação extraterrestre (Ra), apesar de ter apresentado o melhor desempenho, não proporcionou uma melhoria significativa no ajuste de $\mathrm{Rn}$ em relação ao modelo que utiliza somente Rs como variável dependente. Os resultados encontrados com este modelo foram similares aos obtidos com a Eq. 3, com índice "c", coeficiente de variação (Tabela 1) e relação entre valores medidos e estimados (Figura 1D) praticamente idênticos. Esse resultado pode ser atribuído à radiação extraterestre (Ra) ter contribuição no cômputo da radiação solar global (Rs), ou seja, o efeito da Ra está intrínseco nos dados medidos de Rs.

$\mathrm{Na}$ validação dos resultados, baseada na série com 360 observações (Tabela 2), apesar do desempenho ter sido inferior, os modelos mostraram o mesmo comportamento já verificado, isto é, as Equações 3 e 4, que utilizaram Rs como variável independente, tiveram o melhor desempenho (índice " $c$ " superior a 0,80), seguidas da Eq. 2 (índice "c" de 0,59) e da Eq.1, que novamente apresentou a performance mais desfavorável (índice "c" inferior a 0,40 ).

Tabela 2. Validação dos modelos de estimativa da radiação líquida ( $\left.R n, M J m^{-2} d^{2} a^{-1}\right)$ com base no coeficiente de determinação $\left(R^{2}\right)$, índice de concordância de Willmott (d) e índice "c"

\begin{tabular}{|c|c|c|c|c|c|}
\hline Equação & $\begin{array}{c}\text { Rn média } \\
\text { (MJ } \mathrm{m}^{-2} \text { dia-1) }^{-1} \text { ) }\end{array}$ & $\mathrm{CV}(\%)$ & $\mathbf{R}^{2}$ & d & c \\
\hline (1) & 11,1 & 15 & 0,31 & 0,62 & 0,35 \\
\hline (2) & 10,9 & 25 & 0,51 & 0,82 & 0,59 \\
\hline (3) & 10,3 & 35 & 0,78 & 0,93 & 0,82 \\
\hline (4) & 10,4 & 33 & 0,79 & 0,94 & 0,84 \\
\hline
\end{tabular}

Devido ao desempenho ótimo, similar ao da Eq. 4, e à maior praticidade, utilizou-se a Eq. 3 para expressar o modelo Priestley-Taylor como função da radiação solar global (Rs). Assim, substituindo-se na Eq. 6 Rn pela expressão 3, considerando-se $\alpha=1,26$ e G =0, obteve-se a Eq.9, que possibilita estimar $\mathrm{ET}_{0}$ para a região de Dourados a partir de Rs e do fator $\mathrm{W}$, função da temperatura média do ar (Eqs. 7 e 8):

$$
\mathrm{ET}_{0}=0,225 \mathrm{~W} \text { Rs }
$$

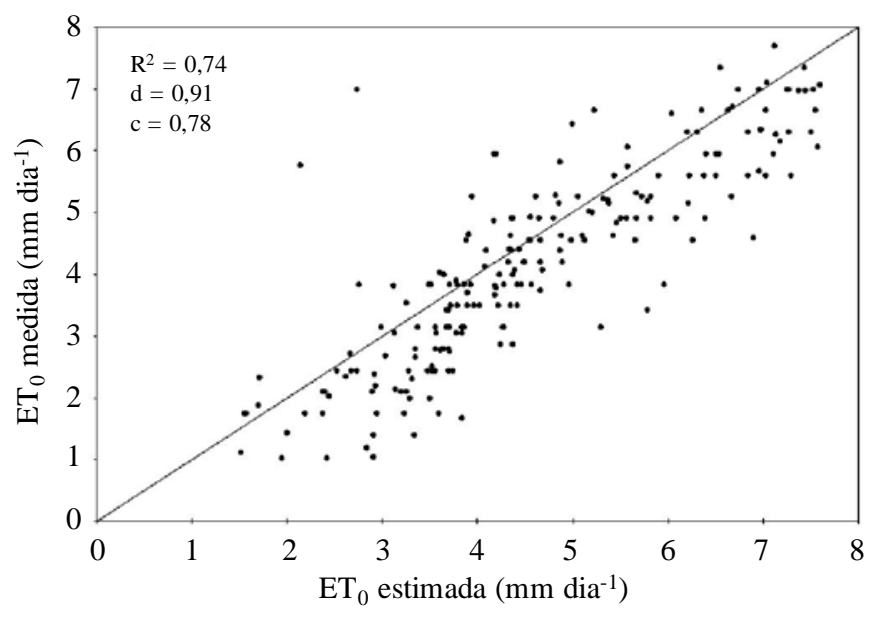

Figura 2. Relação entre evapotranspiração de referência $\left(E T_{0}\right)$ medida e estimada pelo método Priestley-Taylor

O desempenho da Eq. 9 na estimativa da $\mathrm{ET}_{0}$ foi muito bom, com índice "c" de 0,78 (Figura 2). No entanto, pode-se perceber a tendência da Eq. 9 superestimar $\mathrm{ET}_{0}$, com a maior parte dos pontos localizada abaixo da reta 1:1. Esse resultado pode ser atribuído a aproximadamente $70 \%$ dos valores de $\mathrm{ET}_{0}$ serem do período chuvoso de Dourados (primavera e verão). Nessa condição de umidade o valor mais apropriado do parâmetro de Priestley-Taylor para períodos úmidos é significativamente inferior ao valor médio $(1,26)$ geralmente adotado (Sentelhas et al., 2000). Além disso, o fluxo de calor do solo não foi considerado na Eq. 9, o que tende a aumentar a superestimativa de $\mathrm{ET}_{0}$.

\section{CONCLUSÕES}

1. As estimativas do saldo de radiação geradas com base apenas nas temperaturas máximas e mínimas não foram satisfatórias.

2. A equação que utilizou as temperaturas extremas e a radiação extraterrestre na estimativa do saldo de radiação apresentou performance mediana.

3. Os modelos que utilizaram a radiação solar global na estimativa do saldo de radiação da região de Dourados tiveram desempenhos similares, classificados como ótimo. Por sua maior praticidade, a equação que utiliza apenas a radiação solar global como variável independente é mais recomendada para ser utilizada na estimativa do saldo de radiação.

4. Apesar da tendência de gerar valores superestimados, o modelo Priestley-Taylor, expresso como função da radiação solar global, teve desempenho classificado como muito bom, possibilitando estimar a evapotranspiração de referência diária da região de Dourados a partir de dados de radiação solar global e temperatura média do ar.

\section{LITERATURA CITADA}

Allen, R. G.; Pereira, L. S.; Raes, D.; Smith, M. Crop evapotranspiration: guidelines for computing crop water requirements. Rome: FAO, 1998. 300p. (Irrigation and Drainage Paper, 56). 
Camargo, A. P. de; Sentelhas, P. C. Avaliação do desempenho de diferentes métodos de estimativa da evapotranspiração potencial no Estado de São Paulo, Brasil. Revista Brasileira de Agrometeorologia, v.5, n.1, p.89-97, 1997.

Daley, R. Atmospheric data analysis. Cambrigde: Cambrigde University Press, 1991. 455p.

Fietz, C. R.; Fisch, G. F. O clima na região de Dourados, MS. Dourados: Embrapa Agropecuária Oeste, 2008. 32p. (Documentos, 92).

Fietz, C. R.; Silva, F. C. da; Urchei, M.A. Estimativa da evapotranspiração de referência diária para a região de Dourados, MS. Revista Brasileira de Agrometeorologia, v.13, n.2, p.250-255, 2005.

Galvão, J. A. da C.; Fisch, G. F. Balanço de radiação em área de pastagem na Amazônia. Revista Brasileira de Agrometeorologia, v.8, n.1, p.1-10, 2000.

Pereira, A. B.; Sentelhas, P. C.; Villa Nova, N. A. Estimativa do balanço de energia radiante em função de elementos climáticos. Revista Brasileira de Agrometeorologia, v.6, n.2, p.201-206, 1998.

Pereira, A. R.; Angelocci, L. R.; Sentelhas, P. C. Agrometeorologia: Fundamentos e aplicações práticas. Guaíba: Agropecuária, 2002. 478p.

Pereira, A. R.; Villa Nova, N. A.; Sediyama, G. C. Evapo(transpi)ração. Piracicaba: FEALQ, 1997. 183p.

Priestley, C. H. B.; Taylor, R. J. On the assessment of surface heat flux and evaporation using large-scale parameters. Monthly Weather Review, v.100, n.2, p.81-92, 1972.
Sentelhas, P. C.; Nascimento, A. L. C. Variação sazonal da relação entre o saldo de radiação e a irradiância solar global. Revista Brasileira de Meteorologia, v.18, n.1, p.71-77, 2003.

Sentelhas, P. C.; Pereira, A. R.; Folegatti, M. V.; Pereira, F. A. C.; Villa Nova, N. A.; Maggiotto, S. R. Variação sazonal do parâmetro de Priestley-Taylor para estimativa diária de evapotranspiração de referência. Revista Brasileira de Agrometeorologia, v.8, n.1, p.49$53,2000$.

von Randow, R. C. da S; Alvalá, R. C. dos S. Estimativa de radiação de ondas longas atmosféricas no pantanal Sul Matogrossense durante os períodos secos de 1999 e 2000. Revista Brasileira de Meteorologia, v.21, n.3b, p.186-200, 2006.

Willmott, C. J. On the validation of models. Physical Geography, v.2, p.184-194, 1981.

Zanetti, S. S.; Sousa, E. F. de; Bernardo, S.; Posse, R. P.; Duarte Jr, J. B.; Reis, F. O.; Detmann, E. Estimação da radiação líquida em função das temperaturas máxima e mínima do ar na região de Campos dos Goytacazes. In: Congresso Brasileiro de Engenharia Agrícola, 33., 2004, São Pedro.Anais ... Campinas: FEAGRI/UNICAMP: Embrapa Informática Agropecuária, 2004. CD-Rom

Zanetti, S. S.; Sousa, E. F.; Bernardo, S.; Posse, R. P.; Melo Jr, J. C. F. de. Estimação do saldo de radiação e da evapotranspiração de referência, em função das temperaturas máxima e mínima do ar. Revista Brasileira de Agrometeorologia, v.12, n.1, p.134-139, 2006. 